\title{
A Soft Computing Modeling to Case-based Reasoning Implementation
}

\author{
Salama A. Mostafa \\ College of Graduate Studies \\ Universiti Tenaga Nasional \\ Kajang, Selangor, Malaysia
}

\author{
Mohd Sharifuddin Ahmad \\ College of Graduate Studies \\ Universiti Tenaga Nasional \\ Kajang, Selangor, Malaysia
}

\author{
M. A. Firdaus M. \\ College of Engineering \\ Universiti Tenaga Nasional \\ Kajang, Selangor, Malaysia
}

\begin{abstract}
Soft computing is a field of computer science that studies the possibility of finding new models to deal with cognitive functions' problems. Such problems can cover but are not limited to perception, systematic thinking, reasoning, object recognition, data mining, episodic memory, control, and knowledge management. The techniques that are normally utilized to establish such models are Fuzzy Logic (FL), Artificial Neural Network (ANN), and Genetic Algorithm (GA). Nonetheless, compared to these techniques, CaseBased Reasoning (CBR) has the potential to host diverse combination of techniques in its architecture. However, constructing an efficient CBR system is still a controversial research issue. Many approaches based on Soft Computing (SC) methodology have been developed to serve multidisciplinary and demanding engineering domains. This paper discusses some of these approaches that utilize various SC techniques in CBR systems. It proposes a new framework which can be adopted in many applications that requires Computational Intelligence (CI) solutions. The framework is built under the concepts of SC where FL, ANN and GA are exploited to perform soft case-based reasoning tasks. The paper's target is to encourage and help researchers in investigating the matter further
\end{abstract}

\section{General Terms}

Cognitive functions, approximate reasoning, uncertainty, pattern recognition.

\section{Keywords}

Computational Intelligence (CI), Soft Computing (SC), CaseBased Reasoning (CBR), Fuzzy Logic (FL), Artificial Neural Network (ANN), Genetic Algorithm (GA).

\section{INTRODUCTION}

There is no significant difference between the Computational Intelligence (CI) branch of Artificial Intelligence (AI) and the concept of Soft Computing (SC). Both of them share the same numerical AI techniques and have somehow quite close meaning [1]. In general, the focused scope under which SC works is the CI. As a result, there is no significant definition that can specifically draw the boundary of each. A survey to investigate what CI is, Duch [2] came up with this definition: "computational intelligence is a branch of computer science studying problems for which there are no effective computational algorithms. It includes the study of all nonalgorithmizable processes that humans (and sometimes animals) can solve with various degree of competence." Duch [2] generalized the definition by not limiting to specific category of a domain and subjecting to specific techniques to avoid restricting the types of techniques used. Soft Computing explores new disciplines with the aim of constructing new generation of AI [3]. The newly constructed intelligent model is capable of providing solutions to the real world problems which are unsolvable mathematically by the traditional hard computing approaches. In this model, approximation and partial truth handling techniques are employed in uncertain and vague situations [1].

The techniques employed under the umbrella of soft computing are divided according to their usage in two approaches: approximation and optimization. They include, but are not limited to, Fuzzy Computing, Probabilistic Computing, Neural Computing and Evolutionary Computing [4]. The environment that must be situated to develop soft computing model is to be in the complementary state. As such, each technique contributes a distinct methodology for addressing a problem in its domain [5]

As mentioned earlier, SC platform consists of several numerical-based AI techniques (i.e. hybridized model) that work together to give a solution to a problem that does not have specific border and is difficult to be modelled mathematically. The first and the most successful hybrid approach is the incorporation of Fuzzy Logic (FL) and Artificial Neural Network (ANN) in Neuro-fuzzy Systems (NFS) [6].

This idea of NFS hybridization originates from two observations: FL is mainly concerned with imprecision and approximate reasoning. It is neither capable of learning, adaptation, curve-fitting nor parallel computation, whereas these characteristics are clearly attributed to ANN. In contrast, ANN is a probabilistic reasoning technique with uncertainty and belief propagation. Its weaknesses are observed in the lack of flexibility, human interaction, interpretability or knowledge representation, which lies at the core of FL [1].

Similar arguments were used to generate other hybrid approaches like Genetic-Fuzzy Systems, the main aim of which was to join the robust search capabilities of Evolutionary Computing and the properties of FL. Evolutionary Computing (EC) is a type of computing methodology with which problem solving methods are applied based on the principles of biological evolution, for instance, genetic inheritance and natural selection. The applications of such techniques are increasingly popular to solve a wide range of problems. The impact of EC is in the practical applications ranging from industry and commerce to the leading edge scientific researches [7].

However, SC is a model with implicit integration machinery to cope with problems that are not within the scope of the 
more traditional hard computing techniques. Fig. 1 describes $\mathrm{SC}$ system main contents.

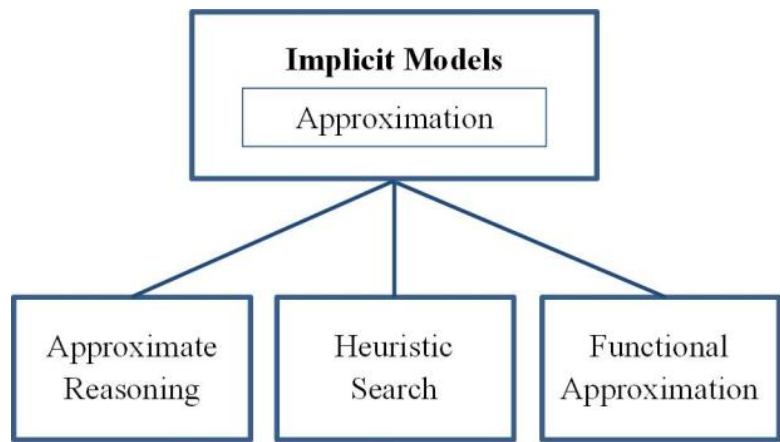

Fig 1: Soft computing [8]

The SC combination shows a way of processing problems when a precise model is either unapproachable or unaffordable due to its complexity.

\section{REASERCH OBJECTIVES}

There are many applications in which Case-Based Reasoning (CBR) integration with the soft computing techniques are made. In this research, a brief description to such integration is made to assist the reader gain some insights into the scope of soft CBR applications. The objectives of the research are as follows:

- To list out some possible combinations of CBR with soft computing techniques.

- To propose a framework that can provide soft CBR approach based on the reviewed combinations.

\section{CASE-BASED REASONING}

Case-Based Reasoning (CBR) is an AI technique that has reasoning ability by remembering previously experienced cases. For computer reasoning purposes CBR system has been formalized into four main steps: Retrieve, Reuse, Revise and Retain. All the four steps are utilized to control data flow of the CBR to produce reasoned result (see Fig. 2) [9], [10]. It records and documents cases and then searches the appropriate cases to determine their usefulness in solving new cases presented to the expert.

The case's main components are the problem (or its symptoms), the solutions, and annotations about the solution derivation. The following gives brief descriptions of the main steps in CBR architecture [9], [10]:

Step1- Retrieve: When a new case arises, the CBR retrieves the most similar case or group of cases to the given problem from the case base.

Step2- Reuse: In this step, the information, knowledge, and solution in the retrieved case or cases are used to solve the target problem if there is a perfect match. This usually involves adapting the solution as needed to fit the new circumstance.

Step3-Revise: If a perfect match is not found having mapped the previous problem to the target situation, revising and adapting the most similar case or group of cases as appropriate are made in this step. The revision of the solution needs to be verified to ensure its acceptance. The verification method used however, depends on the model structure, the application itself, and the way the revision is performed.
Step4- Retain: After the solution has been successfully adapted to the target problem, the new experience or resultant case is saved as a new case in the case base. The case base is updated by saving the newly learned case to be utilized in the next problem solving cycle. In the retain step however, there might be unlearning process involved to prevent out-dated or obsolete cases in the case base.



Fig 2: CBR cycle

From Fig. 2, there are two possible cycles in the CBR. The first one, marked 1, represents an existence of a similar case in the case base and only the retrieve and reuse steps are required. While in 2, the CBR performs all the four steps in an incomplete match solution cycle.

In the present work, the retrieval process is performed on the basis of cases similarity measurement to determine cases classification which is handled by FL. ANN is used in the revision step of the CBR cycle by processing the collected data from the retrieve step and finding the solution. The solution is found based on the most similar cases analysis. Then the process of the solution retaining step is delegated to the GA.

\section{WHY CASE-BASED REASONING}

$\mathrm{CBR}$ as a field deals with both theoretical and practical problems to convey reliable and plausible reasoning. It has been affected by a number of other fields, for instance, cognitive science, knowledge-based systems, machine learning, databases, information retrieval, fuzzy logic and neural networks [11]. There are also a lot of commonalities with other fields such as uncertainty, pattern recognition, and statistics. Fig. 3 depicts the main fields influencing the CBR [12].

$\mathrm{CBR}$ is an open domain for integrating and combining various types of techniques. It is right to mention that many hybrid systems incorporate the use of CBR. Some examples and supporting works are [12], [13], [14], [15], [16], [17] and [18]. CBR has all the potentials of a complete AI framework by having the ability to easily adopt other AI techniques in its reasoning cycle [9]. It can deal with both computational and knowledge base problem solving intelligence [19]. 


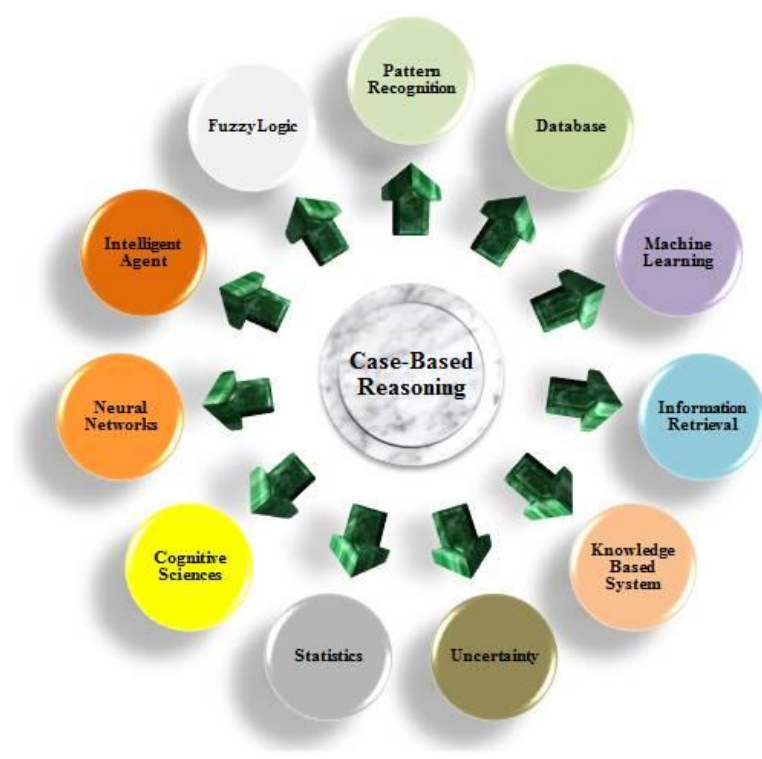

Fig 3: Main fields influencing the CBR

The traditional AI techniques tend to solve incidents from first principle (fundamental bases) while CBR systems produce solutions to the new incidents by using the knowledge of past experiences [20]. It can use solutions that are stored in its case base to solve similar repetitive problems which reduce the solution cost. Solution validation cost is also saved as a result of its previews availability. Furthermore, if there is no similar case in the case base, generating and verifying the solution in the revision step is established based on the experiences of the existing case which presents a starting milestone instead of having to start from scratch, especially in applications where data are too scanty [21].

Knowledge growth in CBR supports incremental, sustained learning. After CBR solves an incident, it makes the incident available for future incidents (i.e. the retain step). Unlike other AI techniques, CBR system's expertise resides in a case library (i.e. the case base and the general knowledge), rather than being encoded in the form of rules. This helps the knowledge and the reasoning process to be implicit in the solution or explicitly recorded as a reused component (this is known as "derivational analogy") [9]. Finally, CBR advantages excel in complicated situations such as when the required rules are difficult to formulate and when the cases' solutions are readily available but the domain expert is not available, unreliable, uncooperative, or inconsistent [22].

\section{CBR COMBINATION WITH SC TECHNIQUES}

In many situations, the AI techniques that are applied to a particular system need not be exclusive alternatives to each other but can be seen as complementary tools that can be brought together within a hybrid system. In addition, there is a wide variety of combination techniques that can be applied to particular problems. Such systems include knowledge-based systems, computational intelligence techniques, and conventional programs [19].

CBR is a popular technique that is capable of exploiting the stored knowledge to handle similar incidents. Combinations of CBR with other intelligent techniques have been explored, deriving effective knowledge representation schemes. Although some types of combinations have been mostly explored, other types have not been thoroughly investigated. $\mathrm{CBR}$ as a framework has the flexibility to combine with other intelligent techniques effectively [23]. The combinations can either involve embedded approaches in which the CBR is the primary intelligent method that embeds one or more intelligent methods to assist its internal online and offline tasks or other possibilities, that is, a collection of AI techniques including externally connected CBR to perform tasks for which different representation formalisms are required or available.

The popular AI techniques' combinations in which CBR represents the key aspect involves combinations with soft computing methods, model-based reasoning (MBR), rule based reasoning $(\mathrm{RBR})$, and other intelligent methods like ontologies [24]. Moreover, CBR can be combined with multiple intelligent systems in multi-integrated paradigms. Distinctive examples of utilizing multiple soft computing methods within the CBR cycle are presented in [25], [26] and [27].

In [25], each step of the CBR cycle is represented by one or more of the soft computing methods. Specifically, ANN is used in the retrieve step, the reuse step is applied using radial basis neural network, the revise step is implemented using Fuzzy Logic system and the combination of the three performs the retain step. In [26], non-embedded coupling approaches are applied. CBR system is combined with Neurosymbolic method in the reconciliation coupling approach [24]. Some of the CBR and soft computing combinations methods are elucidated in the following subsections.

Wen-der Yu and Yan-ching Liu [27] propose a new data mining technique from the hybridization of both numeric and symbolic reasoning techniques and investigate the higher mining accuracy by examining several combinations including CBR with ANN and NFS. The experimental result shows that CBR and NFS hybridization score the highest accuracy. The system framework of the proposed Hybrid CBR by [27] is outlined in Fig. 4.

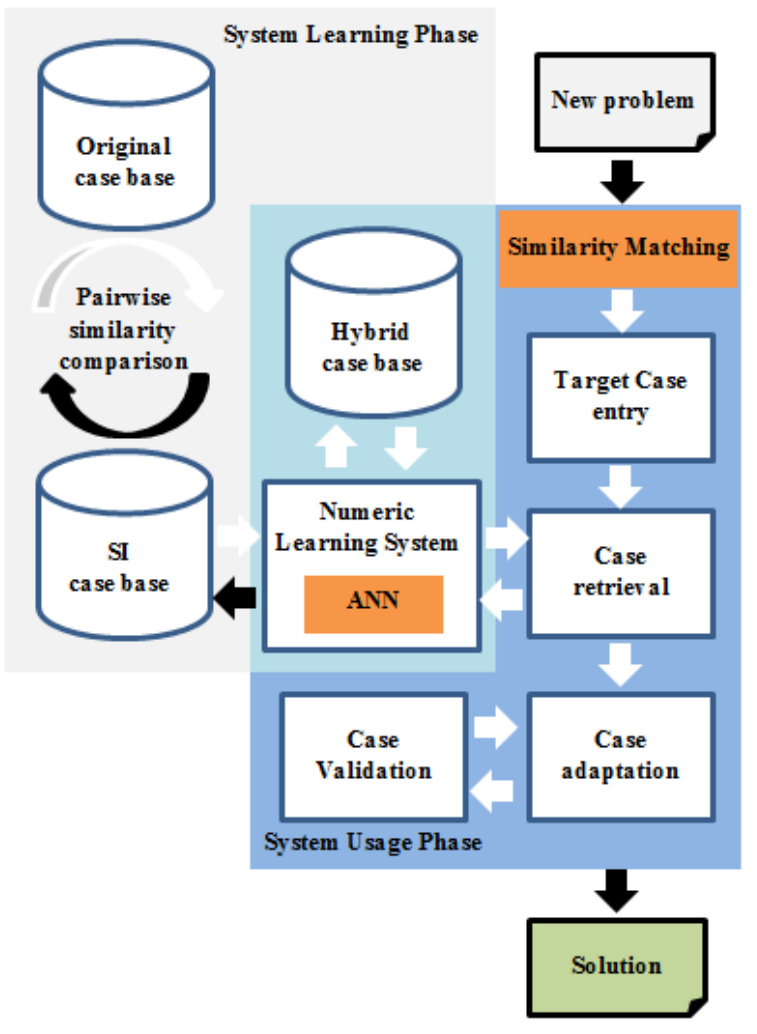

Fig 4: Generic framework of hybrid CBR 


\subsection{Combinations of CBR with FL}

$\mathrm{CBR}$ in some states tolerate uncertainty and imprecision and managing both are of high importance in ensuring accuracy and precision [28]. This is dependent on the applications and technology used within the CBR. The sources of uncertainty and imprecision in CBR are: insufficient information required for decision making, different contexts and respective information that give different meaning, and poor definition of goals and objectives by the user. Moreover, CBR as a system encounter indistinct mechanism that can justify the decision made [29]. As a result, uncertainty and vagueness are inherent in various CBR tasks.

The use of Fuzzy Logic (FL) in CBR cycle for different purposes is very common. FL can be applied in CBR system for imprecision handling. The incorporation of FL in a CBR system leads to improve the overall system performance and eliminate some of its deficiencies [30]. The possible employment of FL in the CBR system can take place as follows:

- In case representation: Fuzzy representation of the design case assists in constructing precise features to the cases from the provided information, consequently the retrieve phase [31].

- In case indexing and retrieval: Assigning degree of membership to the cases' features to be selected based on specific criteria [30]. Consequently, it can assist in cases similarity measurement and matching mechanism as in [32] and [33]. The Aggregation of both improves the retrieval results and deciphering the imperfect knowledge [34].

- In case adaptation: Fuzzy rules can be utilized to distribute the solution in such a way that can lead to problem resolution [23]. The membership functions of FL are responsible for performing the adaptation by manipulating the values of the respective parameters of the retrieved cases [35].

- In revision: Providing solutions to uncertain or incomplete cases [25]. Revision might require combinations of methods rather than one method as a result of its complexity.

Case base maintenance: FL can be employed to eliminate redundant or contradictory solutions in the CBR case base. As the new case needs specific representation and indexing the revised solution requires the same treatment [9]. In this matter, FL can almost play the same role in the retrieve step. In addition, cases matching and similarity measurement is also required to maintain the case base. However, this type of employment is costly and may require a lot of operators to handle the volume of the new cases [6].

\subsection{Combinations of CBR with ANN}

In some situations of insufficient knowledge, Artificial Neural Network (ANN) performs better than CBR especially when it is difficult to express data in a symbolic representation, for example, in network intrusion detection where ports, addresses, protocols, etc. are in numeric form. Their field of application leans towards pattern recognition (i.e. when a lot of points are provided as raw data e.g. vision, speech, image processing). Due to the fact that there are many types of ANNs that have been developed, for instance, Single-Layer Perceptron (SLP), Multi-Layer Perceptron (MLP), Radial Basis Function (RBF) and Hopfield networks, their capabilities for performing tasks like clustering, prediction, and classification are also different [19].

Recently, there are many applications that are built with the combination of the CBR and the ANN (e.g. [25], [26]) in which ANN performs various internal CBR tasks. ANN is usually employed by CBR to perform tasks related to its reasoning step. For instance, ANN can be employed to extract new knowledge from the existing one to be exploited by CBR. As such, the ANN characteristics of parallelism, adaptability, generalization, robustness to incomplete data handling are practically used [30]. The following are possible applications of ANN in CBR systems.

- In case retrieval: ANN is very tolerant in dealing with incomplete data with an acceptable performance in retrieval. For better performance however, the coverage of the ANN domain has to be exhaustive during the learning phase. In view of these advantages, ANN and CBR integration cooperate to handle sophisticated problems, for instance, the PATDEX system [36], [37]. Clustering the available cases in the numerical CBR is another implementation to the ANN in the CBR retrieval as in [9].

- Performing adaptation: Since, CBR adaptation ability deteriorates if there are no exact similar cases, ANNs such as MLP and RBF can be applied in the CBR adaptation. In [38] for instance, ANN is used for parameter adaptation to predict solutions to unfamiliar cases. Likewise, MLP with one hidden layer was developed for this purpose using back-propagation algorithm for training. [35]. Yet, RBF has the advantage over MLP in the situation of poor number of available cases.

- Case feature weighting: the ANN can be utilized to learn the global feature weights (i.e. assigning weights to the case features) [32]. This can assist in case similarity measurement in the retrieve and reuse steps. In essence, feature weights show priority/importance and thus, the relation of the available features.

\subsection{Combinations of $\mathrm{CBR}$ with GA}

Genetic Algorithm (GA) integration in CBR system is for optimization purposes, which can be utilized in different aspects. Some of the possible utilizations of GA in the CBR system are as follows:

- Indexing and retrieval optimization: In the case similarity measurement, GA can be used to generate and assign weights to the cases' features [10], [39]. This can help in determining features and case selection and retrieval with a minimum predictive error [40]. In addition, instance selection results from this process by reducing the search space and finding the optimal cases to be retrieved [41].

- Case adaptation enhancement: In situations of many similar case solutions, GA can be utilized to select the best case among them or it can select the best features from the given cases to be presented as a new case to be used (i.e. case feature discretization) [42]. Another technique is proposed by [43], the GA population is initiated from the retrieved data, mutation and crossover operators are utilized to generate the required population and the fitness function evaluates the generated solution.

- Parameter adjustment: It is another approach that is used in case adaptation enhancement. GA is employed to 
determine the amount of adjustment needed to modify the solution [6].

- General purpose: It is more convenient to enable the GA to work on more than one aspect of the CBR than one particular problem (e.g. [40], [41] and [44]). In [44], GA is utilized to generate useful cases of the proposed CBR module. Besides, it also handles various steps of the CBR module such as evaluation and retrieval, adaptation, and learning for refining the module performance. For case evaluation and retrieval enhancement, a fitness measure of a case that is based not only on the combination of its features values, but also on it being a member of the case base, is proposed.

The synergy between CBR and GA can take another form. CBR can be employed for enhancing GA performance by enabling it to work in a dynamic mode. In this case, the chromosome of the GA can be represented in case form with the privileges of the CBR's four manipulation steps as in new chromosomes generation and periodical injection as described in [45]. Moreover, in the GA learning, several concepts can be applied with the assistance of the CBR such as meta-genetic code, injection, breakthrough, population migration, refinement, and deterministic mating [44]. In addition, CBR can be utilized to track and record the history of GA during its optimization processes. This can help to improve GA performance by remembering the shortest and the best needed activities and/or strategies used to a particular problem such as remembering the best initial population [46]. In a more complicated approach, CBR can be utilized in GA operators' selection. In this type of GA, multi-operators are applied to find the optimal solution. CBR operators sequence selection is made based on specified attributes.

\section{THE PROPOSED FRAMEWORK}

This paper provides a treatise in a unified framework describing how soft computing methods can be utilized in constructing and maintaining CBR system. The framework is a result of the reviewed possible combinations of CBR and SC that were analysed to find the best integration for each of the SC techniques in the CBR system. Other proposed combinations are ignored due to poor impact, less appropriate, highly specific or the availability of other efficient ways [27], [47].

The proposed framework exploits the conspicuous impact of the SC techniques to build an amalgamated computational entity. The conception of the SC techniques is to augment the four major steps of the problem-solving life cycle of the CBR, specifically, in case indexing, selection and retrieval, case adaptation, case revision, CBR learning and case base maintenance. The result is a soft case-based reasoning which can be utilized in different real world applications.

In the proposed framework, the case is broken down to a number of features. Each feature consists of a number of elements. The elements describe the property of the corresponding feature, while the features describe the overall case. This structure of the case can help in simplifying the processes of the reasoning cycle. Fig. 4 shows an abstract example of the proposed case representation.

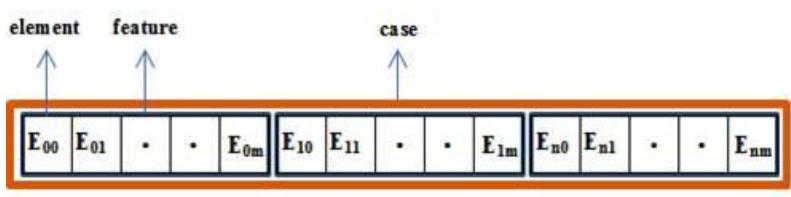

Fig 5: The proposed case representation

In the figure, $\mathrm{E}$ is the element of the feature, $\mathrm{n}$ is the number of features in the case, and $\mathrm{m}$ is the number of elements of the corresponding $\mathrm{n}$ feature. To avoid system complexity, $\mathrm{n}$ and $\mathrm{m}$ are to be equal in all the cases. When a problem takes the system's case form and the features are obtained, searching of similar cases in the case base is performed.

The search algorithm used is designed for the application that it is situated in. Similarity measurement of both cases and features is then made and each component weight is consequently obtained. The cases with the highest rate of weights are retrieved as relevant cases. Then FL classifies the relevant cases into complete-match, partial-match, and nomatch cases based on the membership that each case has with the problem case. All the above are exploited in the retrieve step shown in Fig. 6. 


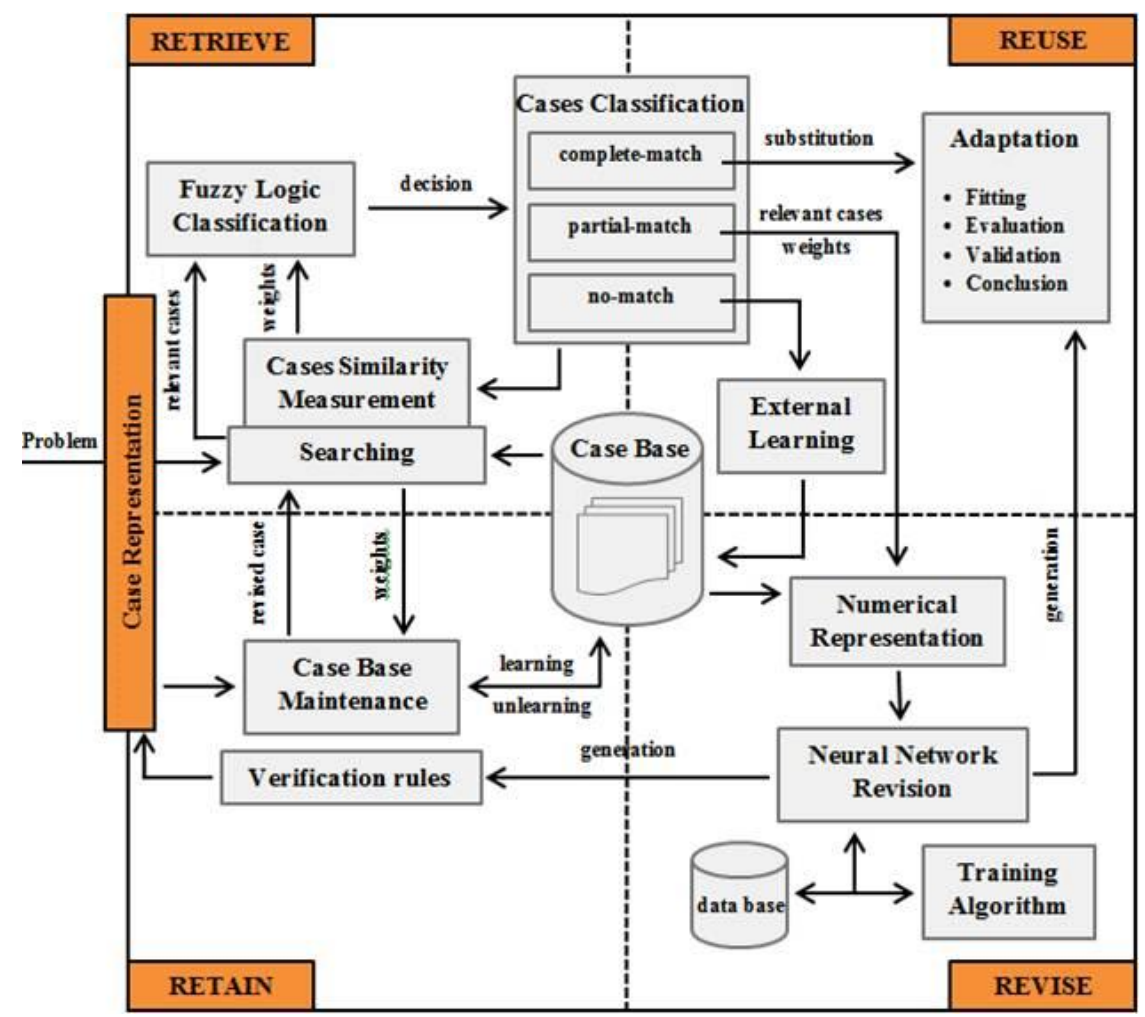

Fig 6: The proposed soft CBR framework

If there is a complete-match, the solution is adapted to solve the problem. The adaptation might require some changes to the case solution to satisfy the problem. The solution is then used and the result is evaluated and validated. The collected information during the adaptation is added to the solution case. If there is no-match, external sources are needed to solve such problems and the solution is stored in the case base to be matched and used. Finally, if there is a partial-match, the partial-match cases and the obtained weights are numerically formatted to prepare for the revision.

ANN is proposed in the revise step to generate solution to the semi-familiar incidents. The solution is generated based on the partial-match relevant cases and the weights of these cases which are found during the retrieve step. The partial-match cases are the input to the ANN (they might need clustering operation) and the weights of the features can play a role in the ANN weights and bias setting. The ANN has to be trained to handle such tasks. The proposed training utilizes the available cases as examples with fixed corresponding outputs. We do not specify the type of ANN used because its type selection depends on the application's needs. In addition, the ANN training algorithm selection depends on the ANN type [48]. However, the ANN has the ability to learn associations between input vectors and associated outputs. The underlying reasons for the associations are unclear. These associations are effectively encoded in the weightings on the interconnections between the neurons. Efforts have been made to automatically extract, from the network, equivalent rules that can be readily understood and to write verification rules to check the validity of the network's output [19].

The generated solution during the revise step is adopted after ensuring its correctness. Reliance on the output from the ANN without any means of verification is not acceptable. It has, therefore, been proposed that rules have to be used to verify that the ANN output is consistent with its input [48]. Neural networks are good at interpolation (i.e. finding solutions by constructing new data from the given data) but poor at extrapolation when the solution is out of the given data scope [19]. One solution to the extrapolation problem in ANN, however, is to train it again to overcome the unfamiliar data and to have better fitting.

The retain step in the proposed framework has a case base maintenance system. This system can be handled by different techniques such as GA. The reason behind applying such system is to reduce storage redundancy as well as improve retrieval efficiency [6]. The revised case is to be checked and learned by retaining it in the case base. As a part of case base maintenance, the less useful case is to be eliminated during the unlearning period. Less useful case selection is made based on the case similarity measurement that is held between the revised case and the available cases. Usability parameters can also be utilized in determining the less useful cases. Such parameters contain information about each case state (i.e. number of time used, time of usage, and similarity level). As a result, the non-useful case is replaced with the revised case.

\section{CONCLUSION AND FUTURE WORK}

The soft computing techniques that are utilized in the CaseBased Reasoning (CBR) system application show observed improvement such as in the multiple selection criteria handling, case retrieval accuracy, and system adaptation ability. Use of the membership function in the retrieve step enhances case selection. The incorporation of Artificial Neural Network (ANN) leads to better generalization by exploiting its learning advantages which enhances the performance of case adaptation. The appropriate combinations of techniques in any system depend on the type of problem and the available data. 
The soft computing objective is to develop models that can be utilized in solving problems in an intelligent way whenever there is no existing other ways to solve the problems or the existing ways are not good enough. The proposed framework is a good option for complex systems that might have nonlinear, time-variant and/or ill-defined cases. Moreover, its usefulness is in situations where the option of mathematical model is either too difficult to encode, does not exist and/or is too complicated and expensive to be made correctly.

General areas that need this kind of framework encompass: classification, scheduling, data mining, control, prediction, optimization and decision support. It is believed that this paper's idea can contribute significantly in improving the quality of CBR cycle. The combination of soft computing methods used can stabilize the level of guessing and approximation through CBR additional learning properties.

The proposed soft CBR employs Fuzzy Logic (FL) and ANN for its performance capability enhancement when FL is utilized to set ANN parameters. With this combination, CBR is converted to a very powerful computational framework. As an addition to this research, a study on the possibility of applying Genetic Algorithm (GA) in the revision part of the proposed CBR cycle is achievable. Moreover, utilizing the framework in a real world application, for instance, object recognition in computer vision application is also being considered.

\section{REFERENCES}

[1] L. Magdalena, "What is Soft Computing? Revisiting Possible Answers," International Journal of Computational Intelligence Systems, vol. 3, No. 2, pp. 148-159, 2010.

[2] W. Duch, "What is Computational Intelligence and Where Is It Going?" Studies in Computational Intelligence, vol. 63, pp 1-13. 2007.

[3] H. R. Enrique, "Soft Computing: Coping with Complexity," International Journal of Computational Intelligence Systems, Vol. 3, No. 2, 190-196, 2010.

[4] P. P. Bonissone, Y. T. Chen, K. Goebel and P. S. Khedkar, "Hybrid Soft Computing Systems: Industrial and Commercial Applications," Proceedings of IEEE, vol. 87, No. 9, pp. 1641-1667, 1999.

[5] I. J. Rudas and J. Fodor, "Intelligent Systems," International Journal of Computers, Communications, and Control, vol. 3, pp. 132-138, 2008.

[6] K. P. Sankar, and C. K. Simon, "Foundation of Soft Case-Based Reasoning," John Wiley \& Sons, Inc., Hoboken. New Jersey, 2004.

[7] A. E. Eiben and J. E. Smith, Ed., "Introduction to Evolutionary Computing," Springer, 2nd ed. ISBN: 9783-540-40184-1, 2007.

[8] S. W. Kercel, "Guest Editorial Special Issue: Industrial Applications of Soft Computing," IEEE Trans. on Systems, Man and Cybernetics-Part C, vol. 36, No. 4, pp. 450-452. 2006.

[9] A. Aamodt, and E. Plaza, Case-Based Reasoning: Foundational Issues, Methodological Variations, and System Approaches: AI Communications, IOS Press, 1994, Vol. 7, No. 1, pp. 39-59.

[10] L. De Ma`ntaras, R. Mc Sherry, D. Bridge, D. Leake, D. Smyth, B. Craw, S., et al., "Retrieval, Reuse, Revision and Retention in Case-Based Reasoning," Knowledge Engineering Review, vol. 20, No. 3, pp. 215-240, 2005.

[11] S. Sathasivam, “Applying Knowledge Reasoning Techniques in Neural Networks," Australian Journal of Basic and Applied Sciences, vol. 6, No. 2, pp. 53-56, 2012.

[12] K. D. Althoff, "Evaluating Case-Based Reasoning Systems: The INRECA Case Study. Habilitationsschrift," Postdoctoral thesis, Department of Computer Science, University of Kaiserslautern, 1997.

[13] A. Aamodt, "Explanation-Based Case-Based Reasoning," In Wess, Althoff \& Richter, pp. 274-288, 1994.

[14] D. W. Aha and J. Daniels, (Eds.), "Case-Based Reasoning Integrations," Papers from the AAAI 1998 Workshop, 1998.

[15] B. Lees, (ed.), "Workshop on Hybrid Case-Based Reasoning Systems," In Schmidt \& Vollrath, 1999.

[16] D. W. Aha, "The Omnipresence of Case-Based Reasoning in Science and Application," KnowledgeBased Systems, vol. 11, No. 5-6, pp. 261-273, 1998.

[17] S. K.. Pal, T. S. Dillon and D. S. Yeung, (Eds.), "Soft Computing in Case-Based Reasoning," Springer Verlag, London, 2000.

[18] A. Aamodt, "Knowledge Acquisition and Learning by Experience-the Role of Case-Specific Knowledge. In Kodratoff and Tecuci (1995), pp. 197-245, 1995.

[19] A. A. Hopgood, Ed., Intelligent Systems for Engineers and Scientists, 2nd Ed., CRC Press, 2001.

[20] P. N. Sakolnakorn, Knowledge Management System Improvement Towards Service Desk of Outsourcing In Banking Business, Master thesis, King Mongkut's University of Technology, North Bangkok, 2007.

[21] D. Doyle, A. Tsymbal and P. Cunningham, "A Review of Explanation and Explanation in Case-Based Reasoning," Technical Report TCD-CS-2003-41, Trinity College Dublin, 2003.

[22] B. U. Haque, R. A. Belecheanu, R. J. Barson and K. S. Pawar, "Towards the Application of Case-Based Reasoning to Decision Making in Concurrent Product Development (Concurrent Engineering), KnowledgeBased System, vol. 13, No. 2-3, pp. 101-112, 2000.

[23] J. Prentzas and I. Hatzilygeroudis, "Categorizing Approaches Combining Rule-Based and Case-Based Reasoning," Expert Systems, Vol. 24, No. 97-122, 2007.

[24] J. Prentzas, I. Hatzilygeroudis, "Combinations of CaseBased Reasoning with Other Intelligent Methods," International Journal of Hybrid Intelligent Systems, ISO Prss, vol. 6, No. 4, pp.189-209, 2009.

[25] F. Fdez-Riverola and J. M. Corchado, "FSfRT: Forecasting System for Red Tides," Applied Intelligence, vol. 21, pp. 251-264, 2004.

[26] K. M. Saridakis and A. J. Dentsoras, "Case-DeSC: A System for Case-Based Design with Soft Computing Techniques," Expert Systems with Applications, vol. 32, pp. 641-657, 2007. 
[27] Wen-der Yu, and Yan-ching Liu, "Hybridization of CBR and Numeric Soft Computing Techniques for Mining of Scarce Construction Databases," Expert System with Applications, vol. 15, No. 1, pp. 33-46, 2006.

[28] A. Idri, A. Abran, T. Khoshgoftaar and S. Robert, "Fuzzy Case-Based Reasoning Models for Software Cost Estimation," In Soft Computing in Software Engineering:,Studies in Fuzziness and Soft Computing, Springer Verlag, pp. 64-96, 2004.

[29] P. P. Bonissone and L. M. Ramon, "F4.3 Fuzzy CaseBased Reasoning System," 2008.

[30] S. C. K. Shiu and S. K. Pal, "Case-Based Reasoning: Concepts, Features and Soft Computing," Applied Intelligence, vol. 21, pp. 233-238, 2004.

[31] K. M. Saridakis, A. J. Dentsoras, P. E Radel, V. G Saridakis, N. V. Exintari, "Neuro-Fuzzy Case-Based Design: An Application in Structural Design," 2006 I*PROMS Virtual International Conference on Intelligent Production Machines and Systmes, 2006.

[32] L. Wang and W. Ip, "A Soft Case-based Reasoning System for Travelling Time Estimation," The Third International Conference on Future Computational Technologies and Applications, pp. 69-74, Rome, 2011.

[33] S. A. Mostafa and H. Mat Jani, "Applying Fuzzy Logic to Software Requirements Specifications Quality Analysis," In The Proceeding of The 2nd International Conference on Advancements in Computing Technology, IEEE/ICACT, Jeju Island, Korea, 2011.

[34] I. El Bitar, F. Belouadha and O. Roudiès, "A Logic and Adaptive Approach for Efficient Diagnosis Systems using CBR," International Journal of Computer Applications, vol. 39, No.15, pp. 0975-8887, 2012.

[35] T. Koiranen, T. Virkki-Hatakka, A. Kraslawski and L. Nystriim, "Hybrid Fuzzy and Neural Adaptation in Case Based Reasoning System for Process Equipment Selection," Computers them. Engng vol. 22, Suppl., pp. S991-S1000, 1998.

[36] K. D. Althoff, and S. Wess, "Case-Based Knowledge Acquisition, Learning and Problem Solving in Diagnostic Real World Tasks," In D. Smeed (ed.), Proc. of the fifth European Knowledge Acquisition for Knowledge-Based Systems Workshop (EKAW-91), Glasgow \& Crieff; 1991, also in: M. Linster \& B. Gaines (eds.), Proc. of the fifth European Knowledge Acquisition for Knowledge-Based Systems Workshop (EKAW‘91), GMD-Studien Nr. 211, pp. 48-67, 1992.

[37] M. M. Richter, "Classification and Learning of Similarity Measures" Proc. 16th Annual Conference of the German Society for Classification, Springer Verlag, 1992.
[38] S. Butdee, "Adaptive Aluminum Extrusion Die Design Using Case-Based Reasoning and Artificial Neural Networks," Advanced Materials Research, Trans Tech Publications Inc, pp. 383-390, 2012.

[39] K. Ju Kim and K. Kim, "Preliminary Cost Estimation Model Using Case-Based Reasoning and Genetic Algorithms," Journal of Computing in Civil Engineering, vol. 24, No. 6, pp. 499-505, 2010.

[40] K. Ju Kim, Toward Global Optimization of Case-Based Reasoning Systems for Financial Forecasting, Applied Intelligence, 21, 239-249, 2004.

[41] H. Ahn and K. Kim, "Global Optimization of CaseBased Reasoning for Breast Cytology Diagnosis, Expert Systems with Applications, Elsevier, vol. 36, pp. 724734, 2009.

[42] K. Ju Kim, S. G. Shih and Y. H. Perng, "Decision Support for Housing Customization: A Hybrid Approach Using Case-Based Reasoning and Genetic Algorithm," Expert Systems with Applications, vol. 31, pp. 83-93, 2006.

[43] G. de Silva Garza and M. L. Maher, "A Process Model for Evolutionary Design Case Adaptation" In Proceedings of the Artificial Intelligence in Design Conference. Dordrecht: Kluwer Academic Publishers, pp. 393-412, 2000.

[44] L. K. Soh and C. Tsatsoulis, "Combining Genetic Algorithms and Case-Based Reasoning for Genetic Learning of a Casebase: A Conceptual Framework," In the Proceedings of the Genetic and Evolutionary Computation Conference, San Francisco, CA, 2001.

[45] S. J. Louis and C. Miles, "Playing to Learn: CaseInjected Genetic Algorithms for Learning to Play Computer Games, "IEEE Transactions on Evolutionary Computation, vol. 9, pp. 669-681, 2005.

[46] E. I. Perez, C. A. Coello Coello and A. HernandezAguirre, "Extraction and Reuse of Design Patterns from Genetic Algorithms Using Case-Based Reasoning," Soft Computing, vol. 9, pp. 44-53, 2005.

[47] I. Hatzilygeroudis and J. Prentzas (Editors), "Combinations of Intelligent Methods and Applications," Proceedings of the 2nd International Workshop, CIMA 2010, France, October 2010. Smart Innovation, Systems and Technologies 8 (SIST-8), Springer, 2011.

[48] P. D. Picton, J. H. Johnson, and N. J. Hallam, "Neural Networks in Safety-Critical Systems," In the 3rd International Congress on Condition Monitoring and Diagnostic Engineering Management, Southampton, UK, 1991. 